\title{
Ghat: An R package for identifying adaptive polygenic traits
}

7 Medhat Mahmoud ${ }^{1,3 q *}$, Ngoc-Thuy Ha $^{2 \&}$, Henner Simianer ${ }^{2,3 \&}$, and Timothy

8 Beissinger ${ }^{1,39 *}$

9

${ }^{1}$ Department of Crop Science, University of Goettingen, Goettingen, Germany

${ }^{2}$ Department of Animal Sciences, University of Goettingen, Goettingen, Germany

${ }^{3}$ Center for Integrated Breeding Research, University of Goettingen, Goettingen, Germany

* Corresponding authors

Medhat Mahmoud (mahmoud1@gwdg.de)

Timothy Beissinger (beissinger@gwdg.de)

"These authors contributed equally to this work.

\&These authors also contributed equally to this work. 


\section{Abstract}

Identifying selection on polygenic complex traits in crops and livestock is key to understanding evolution and helps prioritize important characteristics for breeding. However, the QTL that contribute to polygenic trait variation exhibit small or infinitesimal effects. This hinders the ability to detect QTL controlling polygenic traits because enormously high statistical power is needed for their detection. Recently, we circumvented this challenge by introducing a method to identify selection on complex traits by evaluating the relationship between genome-wide changes in allele frequency and estimates of effect-size. The method involves calculating a composite-statistic across all markers that captures this relationship, followed by implementing a linkage disequilibrium-aware permutation test to evaluate if the observed pattern differs from that expected due to drift during evolution and population stratification. In this manuscript, we describe "Ghat", an R package developed to implement the method to test for selection on polygenic traits. We demonstrate the package by applying it to test for polygenic selection on 15 published European winter wheat traits including yield, biomass, quality, morphological characteristics, and disease resistance traits. The results highlight the power of Ghat to identify selection on complex traits. The Ghat package is accessible on CRAN, The Comprehensive R Archival Network, and on GitHub. 
43

\section{Introduction}

Many important traits in plants, animals, and humans are of polygenic nature and depend on the cumulative effects of many loci, each contributing a small proportion of the total genetic variation [1]. Studying the evolution and selection history of such traits can be extremely challenging due to the small effects of the quantitative trait loci (QTL) that contribute to genetic variation. However, regardless of effect size, the theory of natural selection tells us that alleles with positive effects on fitness will tend to increase in frequency over successive generations [2]. A number of statistical methods have been developed to identify loci carrying such beneficial alleles. These methods were reviewed in [3], and include approaches relying on polymorphism variability, haplotype frequency, linkage disequilibrium, allele frequency change over time, and techniques that combine some or all of these signals. However, in the case of highly-polygenic traits, it is difficult or impossible to identify regions carrying individually-selected alleles/haplotypes due to their small effects, regardless of which sophisticated methodologies are leveraged. For instance, it has been shown that selected loci are difficult to identify when per-locus selection intensity is low [3], as is the case with low heritability traits and those controlled by many alleles of small effect [4]. This imposes serious limitations to the previous methods when identifying selection on polygenic traits (e.g. $[5])$.

Recently, large sample sizes utilized in human genetic studies have allowed more powerful understanding of the genetic architecture of polygenic traits using genome-wide association studies (GWAS) [6-8]. After conceptual work solidified the importance of polygenic adaptation $[9,10]$, researchers began placing more of an emphasis on identifying this phenomenon using an infinitesimal approach [11-14]. For example, Zeng et al recently developed a Bayesian mixed linear model (BayesS) that detected natural selection on 23 complex traits in humans, based on the relationship between SNP effect sizes and minor allele 
frequency [15]. However, it turns out that results from these methods, particularly those relating to selection on human height, are highly sensitive to the confounding effects of population stratification $[16,17]$. Additional methods recently developed to detect polygenic adaptation include SBayesR [18], which is an extension of the Bayesian multiple regression model (BayesR) [19] to utilize the summary statistics from GWAS instead of using individual-level genotype data, and an approach by Josephs et al [20] which performs principal component analysis on the relatedness matrix to detect polygenic adaptation.

Another test, denoted $\hat{G}$ (or Ghat), was recently developed by Beissinger et al [21] to identify selection on polygenic traits. The idea behind this test is to combine information from all loci simultaneously to evaluate whether there is a trend for loci contributing to a phenotype to collectively show evidence of selection. Thereby, signals that are individually insignificant for selection (at the locus level) may become highly significant when evaluated at the genomewide scale (at the level of finding selection on a trait). The test depends on the relationship between changes in allele frequency over time and estimated additive effects for every genotyped locus.

We have developed and released an R $[22,23]$ package called "Ghat" to implement the $\hat{G}$ test. The package allows users to test for selection and evolution in both qualitative and quantitative traits, and as shown by [21], it is particularly powerful when testing for selection on highly polygenic traits. For the purpose of demonstrating the package, in this manuscript we applied the method and corresponding Ghat package to 15 different European winter wheat traits from a collection of 191 wheat cultivars, registered in Europe between 1966 and 2013, which were published by Voss-Fels et al. [24], demonstrating the historical adaptation of one of the most important crops worldwide. 


\section{Implementation of the Ghat Package}

94

95

96

97

\section{Overview}

Ghat identifies selection using all genome markers/sequences information [21]. This makes it possible to identifying selection in complex traits that are controlled by many genes with small effect. First, all allelic effects are estimated using a genomic prediction approach. Next, allele frequencies are estimated in two or more different generations (e.g. generation 0 and 10). Third, the rate of linkage disequilibrium decay is estimated to approximate the number of independent genome segments in the study population. Finally, an LD-aware permutation test is implemented to evaluate if there is evidence of significant selection between generations, and in what direction selection operated.

\section{Installation}

The Ghat package is installed within the R terminal (Fig1, step-1) by typing:

>install.packages("Ghat") and then loading to package into R by typing: >library(Ghat). In addition, the package has the following optional R-package dependencies:

- Parallel-Package \{parallel\}: Support for parallel computation, to speed the computation time in large data analysis. [25]

- $\quad$ rBLUP-Package $\{$ rrBLUP $\}$ : Ridge regression for estimating marker effects (RRBLUP). [26]

Optional dependencies that are not automatically installed with the package, include rrBLUP [26] and parallel [25]. These maybe installed and loaded before running Ghat by typing:

(Fig1, step-1). 


\section{Input Data format}

117 As inputs, Ghat requires two vectors. The first of these is a vector of allele substitution

118 effects, and the second is a vector of the corresponding change in the frequency of the

119 substituted allele between two different generations (Table 1). Required parameters are,

120 "method": to specify how the Ghat permutation test should account for LD between markers

121 ("scale" is typically the most appropriate method to implement), "perms": for the number of

122 permutations to perform, "plot": to specify whether or not simple Ghat plots should be output,

123 "blocksize": for setting the size of blocks that should be used for LD-trimming (only required

124 when method = "trim"), and "num_eff": for setting the effective number of independent

125 segments of the genome (only required when method = "scale"). All parameters details are in

126 Table 1.

127 Establishing the number of effective markers

128 Treating each marker as an independent observation can lead to overestimated test statistics.

129 This is because allele frequencies and effect estimates from markers that are in LD are not

130 independent [21]. Any level of LD between markers produces less independent observations

131 than the total number of markers. In Ghat, we provide a function called "ld_decay" that can

132 estimate the LD decay across all genome and use this information to approximate the total

133 number of independent genome segments, or "genotype blocks". The following command

134 runs the ld_decay function $>$ Ld <- Id_decay(gen, map, max_win_snp, max.chr, cores=1,

135 max_r2) (Fig 1, Step-3). The output information is used by Ghat to scale the variance of the

136 permutation test statistic according to the actual number of independent markers (Fig 1, Step-

$1374)$. 


\section{Running the test}

140 Before running Ghat, one needs to either estimate the allele substitution effects and allele

141 frequencies in at least to different generations, or get that information from some already

142 published analysis i.e. publicly available summary association statistics [27]. To estimate

143 allele substitution effects and allele frequencies, Ghat provides some simple examples to

144 compute both from individual row genotype data. Next, to perform the Ghat test with this

145 information, only one line of code is required: > Ghat_res <- Ghat(effects, change, method,

146 perms, plot, num_eff) (Fig 1, Step-4).

147 Testing for selection using The Wisconsin Quality Synthetic (WQS) data object.

148 To illustrate the use of the Ghat package, we used a previously published maize population

149 dataset (Fig 1) as a case of polygenic adapted species [5]. A very brief summary of the

150 selection protocol is that to improve silage quality, the researchers performed reciprocal

151 recurrent selection using a selection index calculated from multiple traits related to maize

152 quality, including a trait called Acid Detergent Fiber (ADF)

153 (http://www.cornbreeding.wisc.edu). The use of Ghat to analyse selection on ADF in the

154 WQS Maize population can be summarized in four steps, which we depict in Fig 1. 
157 Fig 1. Four steps to go from raw individual marker data to results from the Ghat test for

158 selection. An illustrated example using the "Maize_wqs" data available in the Ghat package.

159 Step-1: install the package, load the dependencies and "Maize_wqs" data and estimate allele

160 substitution effects (e.g., by using rrBLUP [26]). Step-2: calculate the differences in allele

161 frequency between two different generations (cycle 1 and cycle 3). Step-3: estimate the decay

162 of linkage disequilibrium (LD) to calculate the effective number of independent markers.

163 Step-4: calculate the Ghat value and p-value from the permutation test. 
All functions, options and data set available in Ghat are provided in Table 1

Table 1. Objects, data, functions and options found in Ghat package.

\begin{tabular}{|c|c|c|}
\hline \multicolumn{2}{|l|}{ Function } & \multirow{2}{*}{$\begin{array}{l}\text { Description } \\
\text { Input vector of allele substitutional effects. }\end{array}$} \\
\hline \multirow{7}{*}{$\begin{array}{l}\text { Ghat } \\
\text { (Function) }\end{array}$} & effects & \\
\hline & change & $\begin{array}{l}\text { Input vector of changes in allele frequency between two different } \\
\text { generations (could be positive, negative or zero). }\end{array}$ \\
\hline & method & $\begin{array}{l}\text { Input option, "vanilla" (assumes complete linkage equilibrium } \\
\text { between markers), "trim" (excludes markers to approximate } \\
\text { linkage equilibrium some of the extreme values) or "scale" (scales } \\
\text { results to reflect underlying levels of linkage LD). }\end{array}$ \\
\hline & perms & $\begin{array}{l}\text { Input number to set the number of permutations to test for } \\
\text { significance. }\end{array}$ \\
\hline & plot & $\begin{array}{l}\text { Input option to select which plot you need to be returned: "Ghat", } \\
\text { "Cor", or "Both". }\end{array}$ \\
\hline & blockSize & $\begin{array}{l}\text { Input number to set the size of the window on each chromosome } \\
\text { for trimming, only required if method = "trim". }\end{array}$ \\
\hline & num_eff & $\begin{array}{l}\text { Input number to set the effective number of independent variants, } \\
\text { only required if method = " scale". Can be calculated using } \\
\text { "ld_decay" function above. }\end{array}$ \\
\hline \multirow{6}{*}{$\begin{array}{l}\text { ld_decay } \\
\text { (Function) }\end{array}$} & gen & $\begin{array}{l}\text { Input matrix of genotype data. Individuals in rows, genotypes }(0,1 \text {, } \\
\text { 2) in columns. }\end{array}$ \\
\hline & Map & $\begin{array}{l}\text { Input data frame including the name for each marker with a } \\
\text { corresponding }\end{array}$ \\
\hline & max_win_snp & $\begin{array}{l}\text { Input number to set the maximum number of markers allowed per } \\
\text { window within a chromosome before estimating the LD. The } \\
\text { default value is } 2000 \text {. }\end{array}$ \\
\hline & $\max . c h r$ & $\begin{array}{l}\text { Input number to select to which chromosome you want to run the } \\
\text { analysis, chromosomes above this number will be excluded from } \\
\text { the analysis. }\end{array}$ \\
\hline & cores & $\begin{array}{l}\text { Input number to set the optimal number of cores for using } \\
\text { parallelized calculation, since calculation of LD require too much } \\
\text { memory. The default value is } 1 \text { for windows machine. }\end{array}$ \\
\hline & max_r2 & $\begin{array}{l}\text { Input number to set the threshold of correlation between the } \\
\text { neighbourhood variant }\left(\mathrm{r}^{2}\right) \text { to calculate the effective number of } \\
\text { independent variants. }\end{array}$ \\
\hline \multirow{4}{*}{$\begin{array}{l}\text { Maize_wqs } \\
\text { (Data object) }\end{array}$} & Maize_wqs[[1]] & $\begin{array}{l}\text { Data frame, including all SNP names (SNP Names), effects on } \\
\text { Acid detergent fiber (adf) (effects.adf), and changes in allele } \\
\text { frequencies (change) between cycle } 1 \text { and cycle } 3 \text { of selection. }\end{array}$ \\
\hline & Maize_wqs[[2]] & $\begin{array}{l}\text { Data frame, including plant ID's (taxa), estimated breeding values } \\
\text { for Acid detergent fiber (adf), and generation when the phenotyped } \\
\text { strains were grown (year). }\end{array}$ \\
\hline & Maize_wqs[[3]] & $\begin{array}{l}\text { Data frame, map file, each line of the Map file describes a single } \\
\text { marker and contain three columns. 1: Name (SNP id); } 2 \text { : } \\
\text { Chromosome (Chromosome number); 3: SNP position (in base- } \\
\text { pairs). }\end{array}$ \\
\hline & Maize_wqs[[4]] & $\begin{array}{l}\text { Data frame, Genotype (Illumina MaizeSNP50 BeadChip); an } \\
\text { Infinium HD assay (Illumina, Inc. San Diego, CA). 10,017 SNP } \\
\text { markers }(0,1 \text { and } 2 \text { ) after filtration, distributed across the maize } \\
\text { genome [28]. }\end{array}$ \\
\hline
\end{tabular}




\section{Methods}

\section{Testing for selection during 50 years of wheat breeding}

To demonstrate the power and flexibility of the Ghat package, we used it to test for selection during the past 50 years of wheat breeding in Europe. To achieve this, we leveraged a large dataset representing 50 years of commercial European winter wheat varieties that was previously published by Voss-Fels and colleagues [24]. Our analysis methods and results are described below.

Genotypes and phenotypes. A data set of 191 cultivars, registered in Europe between 1966 and 2013, was generated and previously published by [24]. In total, 19 traits were considered and used to evaluate and quantify the phenotypic progress of wheat breeding during the last five decades. However, we used only 15 traits after filtering based on data quality. Spikes per sqm, Heading, Sedimentation, Falling number, and GCD were removed from the analysis because of missing some phenotypes information ( $>70 \%$ of total phenotypes were missing). All traits were tested in three different environments by [24]. The environments were: 1: A high-intensity nitrogen supply along with best-practices fungicide, insecticide, and growth regulator applications (HiN/HiF treatment), 2: A high level of nitrogen fertilization with a fungicide-free treatment (HiN/NoF), and 3: A low level of nitrogen fertilization with a fungicide-free treatment (LoN/NoF). The evaluation was based on five main groups of traits: 1- Yield parameters traits, including: Grain yield, Harvest index, Radiation use efficiency and Radiation interception efficiency; 2- Biomass parameter traits, including: Aboveground Biomass, Kernels per sqm, and Thousand-kernel weight. 3- Quality parameter traits, including: Crude protein, Protein yield and Nitrogen use efficiency. 4- Morphological traits, including: Plant height, Green canopy duration and Kernel per spike. 5- Disease resistances traits, including: Resistance to powdery mildew and Resistance to stripe rust. All cultivars were genotyped with a 15K SNP Illumina Infinium iSelect genotyping array [29] for 
195

196

197

by [24], and after filtering 8,710 SNPs passed the genotype quality control criteria [24] and were included in their subsequent analyses. In our analysis, we re-analyzed the exact set of SNPs that was published by [24], as described above.

Phenotypic progress. As technical validation, we re-estimated the phonotypic progress of the 15 wheat traits over 50 years of selection and adaptation using simple Pearson correlation $[30]$.

Ghat. Allelic effects at every marker locus for the 15 traits were estimated using rrBLUP [26], and changes in allele frequencies were calculated with $\mathrm{R}[22,23]$. Ghat is calculated as the summation of the estimated effect of every SNP scored multiplied by its effect size, according to

$$
\hat{G}=\sum_{j=1}^{m} \Delta_{j} \alpha_{j}
$$

where $\Delta_{j}$ is the frequency change in locus $j$ between two time-points and $\alpha_{j}$ is the additive effect of locus $j$. We implemented two different analyses with Ghat to test for selection, which enabled us to measure the efficiency of the test in different situations. In the first analysis, which we call "All Phenotypes", allelic effects were estimated using phenotypic information from all available cultivars in the Voss-Fels study, which represent the last 50 years of wheat breeding. This represents the best-case Ghat analysis, when all phenotypic information is available. In the second analysis, which we call "Modern Phenotypes", allelic effects were estimated using only modern phenotypic information (from 2010 to 2013), while changes in allele frequency were still calculated based on genotypes from opposite ends of the 50-year dataset. This represents a realistic use-case for Ghat, when the experimenter has all genotypic information, but only phenotypic information from the present. This represents a situation where reliably and comparably measured historical phenotypes no longer exist, but historical tissue or seed are available and can be used for DNA collection. 


\section{Results}

\section{Testing for selection during 50 years of wheat breeding}

The main theory behind this test for polygenic adaptation/selection is that alleles at every locus spread across the genome may contribute a small proportion of the total variance for each trait (infinitesimal model), regardless whether or not a mapping study has enough power to significantly associate markers with traits. To demonstrate the Ghat package, we used it to test for selection on the 15 wheat traits and replicated for two different analysis mentioned above, enabling us to establish how much power is lost when phenotypic data are only available from modern lines.

Phenotypic progress. After estimating the Phenotypic trend (correlation between phenotypic value and the registration year) (Fig 2). We can clearly classify the 15 traits into three groups. The first group is comprised of traits that increased in value during breeding. These were above-ground plant biomass, grain yield, green canopy duration, harvest index, kernels per spike, kernels per square meter, nitrogen use efficiency, protein yield, radiation interception efficiency, radiation use efficiency, resistance to powdery mildew, and resistance to stripe rust. Next, we identified a group of traits with a decreasing phenotypic trend, which was comprised of plant height and crude protein. Finally, thousand-kernel weight showed no significant trend in either direction.

Ghat test for selection. Fig 2 shows the performance of Ghat when testing for selection using genotypes and phenotypic information from lines produced during all 50 years of wheat breeding and comparing it with the actual phenotypic progress of these lines for the past 50

241 years (phenotypic trend). First, we investigated the precision and accuracy of the Ghat in 242 estimating the size and direction of selection (using Ghat-correlation) when all phenotypic information were available (50 years). Overall, the selection size and direction on the 15 traits 
244 using all phenotypes were in the highest agreement with the phenotypic trend. Next, we

245 evaluated Ghat results when the phenotypic information were only available for the past 3

246 years (from 2010 to 2013). Notably, this was also in a strong agreement with the phenotypic

247 trend. Ghat successfully detected the selection history of each trait, even when only modern

248 phenotypes were utilized. For example, Ghat found that Plant height and Crude protein traits

249 were under intensive negative selection, which agrees with their phenotypic trends during the

250 last 50 years. Ghat showed very weak selection for thousand-kernel weight, which again

251 aligned with this trait's phenotypic trend. The rest of the traits were under high to moderate

252 positive selection. To validate the results of Ghat on wheat data, a spearman correlation

253 between the Ghat results from 3 years and 50 years of phenotypes were estimated. A very

254 high significant $(\mathrm{P}<0.001)$ correlation of 0.57 was found between Ghat values from 3 and 50

255 years of phenotypes. 
258 Fig 2. The power of Ghat test of selection in European winter wheat under three

259 agrochemical treatments. 1: A high-intensity nitrogen supply along with best-practices

260 fungicide, insecticide, and growth regulator applications (HiN/HiF), 2: A high level of

261 nitrogen fertilization with a fungicide-free treatment (HiN/NoF), and 3: A low level of

262 nitrogen fertilization with a fungicide-free treatment (LoN/NoF). Left: the phenotypic trend

263 over the last 50 years. Right: the Ghat test of selection based on two analyses. "All

264 Phenotypes" refers to estimating allelic effects using all available phenotypic information

265 over the 50 years of wheat breeding; "Modern Phenotypes" refers to estimating allelic effects

266 using modern phonotypic information only (2010 to 2013). Blue, positive selection; red,

267 negative selection. Large light ellipses correspond to weaker selection intensity; thin dark

268 ellipse correspond to more selection intensity. P-values printed on each cell on the left side of

269 the figure (phenotypic trend section) correspond to the significance of the phenotypic trend.

270 P-values printed on each cell on the right side of the figure (Ghat test section) correspond to

271 implementing the Ghat permutation test. 
273 Adaptation history in the European winter wheat.

274 To more precisely dissect the history of selection in wheat, we divided the 50 years of

275 breeding into 10 adaptation periods and applied Ghat successively between each period. We

276 excluded the green canopy duration trait from this analysis since it was only measured under

277 one environmental condition. For the remaining 14 traits, we calculated the average

278 phenotypic value across the three different environmental conditions. For example, during the

279 period from 1966 to 1975 , significantly selected traits according to Ghat included grain yield,

280 harvest index (Fig 3-A), above-ground plant biomass, thousand-kernel weight (Fig 3-B),

281 crude protein, nitrogen use efficiency (Fig 3-C), kernel per spike and resistance to strip rust

282 (Fig 3-D). However, during the same period a selection operated significantly in the negative

283 direction against radiation use efficiency (Fig 3-A), thousand-kernel weight (Fig 3-B), plant

284 height and resistance to powdery mildew (Fig 3-D). Moreover, by following to the time series

285 of the selection over the past 50 years (Fig 3) we can get a complete picture of how breeders

286 adapted the 191 wheat cultivars through time, by focusing on certain traits in some time

287 periods and other traits in other time periods. 
290 Fig 3. Ghat correlation estimates of the selection size and direction for 50 years of wheat

291 breeding. Results are for 14 productivity traits in European winter wheat measured during the

292 past 50 years. Points above zero level (red dashed lines) indicate positive selection, while

293 points under zero indicate negative selection; bold lines represent 99\% confidence interval. A-

294 Adaptation history for the Yield parameters traits. B- Adaptation history for the Biomass

295 parameters traits. C- Adaptation history for Quality parameters traits. D- Adaptation

296 history for Morphological and disease resistance traits. 


\section{Relative selection pressure}

Relative selection pressure (RSP) can defined as: The average of absolute changes in allele frequency of the alleles that controlling traits. RSP is genetic property of biological traits.

Like heritability, it has a specific value for each trait and varies from zero to one. RSP is a measurement of how allele frequencies of the effective variants changes per year or more under natural/artificial selection [31,32]. We estimated the RSP in this study by calculating the average of all absolute values of Ghat correlation every 5 years. In the case of the European winter wheat data, we estimated the RSP for the past 50 years and we plotted the accumulative selection pressure on these 14 traits in Fig 4. In addition, the actual RSP of the 14 traits listed in Table 2. RSP quantifies how elastic each trait is elastic to the breeding decisions regardless of the selection direction (positive or negative). One notable example of the response to selection in the evolutionary process, is the "Above the ground biomass" trait, with RSP of 0.085 this trait starting from close to zero selection on 1970 to near to 0.75 of accumulative selection. On the other hand, "Thousand-kernel weight" trait did not show high response to selection, since the increase of accumulative selection size did not increased compared to the other traits, and it has the lowest RSP in the list (Table 2)

Table 2. Relative selection pressure (RSP) for 14 traits of European winter wheat. RSP were estimated by calculating the average of the absolute Ghat correlation every 5 years for 50 years.

\begin{tabular}{|l|c|}
\hline Traits & Relative selection pressure (RSP) \\
\hline Above-ground plant biomass & 0.086 \\
\hline Crude protein & 0.085 \\
\hline Grain yield & 0.076 \\
\hline Harvest index & 0.061 \\
\hline Kernel per spike & 0.090 \\
\hline Kernels per sqm & 0.100 \\
\hline Nitrogen use efficiency & 0.079 \\
\hline Plant height & 0.064 \\
\hline Protein yield & 0.079 \\
\hline Radiation interception efficiency & 0.097 \\
\hline Radiation use efficiency & 0.067 \\
\hline Resistance to powdery mildew & 0.069 \\
\hline Resistance to stripe rust & 0.084 \\
\hline Thousand-kernel weight & 0.050 \\
\hline
\end{tabular}


Overall, the average value of RSP in wheat traits is 0.078 (Table 1), the highest was 0.10 in

319 Kernels per sqm, reveals that this traits is most affected traits of in wheat breeding may be because of a direct selection on the traits or a high genetic correlation with other important traits.

Fig 4. Cumulative selection in European winter wheat. Results are for the 14 traits of wheat during the past 50 years ago.

\section{Testing and system requirements}

project.org/package=Ghat) for the three major operating systems: MS Windows, Linux/Unix, and Mac OS. We have also released the source code on GitHub

(https://github.com/timbeissinger/ComplexSelection) so that users can compile for specific

platforms or modify the code as needed.

For users who have already computed allele effect and allele frequencies changes and are applying the Ghat test to these data, the computational cost of Ghat is unnoticeable.

Practically, estimating Ghat for maize data [5] with 1000 permutations takes one second per one phenotype on a 2019 Dell XPS 23 with 2.4 Intel Core i7 processor.

\section{Availability and future directions}

We have presented a new package for identifying selection and adaptation from GWAS or Genomic prediction summary statistics, which we applied to a dataset of Wheat plant after calculating their rrBLUP summary statistics of 15 traits and 119 cultivars phenotype measurements. We reported several new findings that would have been difficult or 
particular loci. These findings including historical adaptation and selection direction on the most important 15 traits of Wheat plant.

A cause of false positive findings in any genome wide analysis is hidden population structure [33]. In additional to the importance of considering population and environmental effects in the estimation model, an effective way to solve this problem is to run principal component analyse and using the most important PC-axis as additional fixed effects in estimation model before running Ghat [34].

Unlike methods for detecting selection, Ghat can quantify selection magnitude and direction even if we do not have any phenotypes from before selection (generation zero). This can be achieve by estimating allele effect by the current population phenotype and estimation allele frequencies from the ancient population by genotyping some ancient tissues that are available in i.e. gene-banks or from other resources. Moreover, we can still approximate allele frequencies from pre-selection generations even without tissue by using current allele frequencies coupled with known pedigree information [35].

For breeders and breeding organizations, Ghat represents a practical solution for tracing selection and adaptation history based on their recorded traits. Furthermore, RSP values can be used to understand how each trait responds to selection and gives breeders an indication of how traits may response to any future selection pressure.

The Ghat test and implementation via the package described herein does not require immense sample size, individual genotypes and/or genome-wide significant SNPs, as demonstrated by our implementation with a relatively modest sample size of 191 wheat individuals genotyped for 8,710 SNPs. Notably, this approach is more powerful when traits are controlled by large number of QTLs (Beissinger et at., 2018). These advantages allow the Ghat package to be used for identifying selection for various complex traits in other species of plants, animals and in humans. The package is available through CRAN (https://cran.r- 
368 (https://github.com/timbeissinger/ComplexSelection).

\section{Acknowledgements}

373 This project was supported with funds from the University of Göttingen, Faculty of

374 Agriculture, Center for Integrated Breeding Research, and Division of Plant Breeding

375 Methodology. We are indebted to the authors of [24] for making their data publicly available. 


\section{References}

1. Fisher RA. XV.-The Correlation between Relatives on the Supposition of Mendelian Inheritance. Earth Environ Sci Trans R Soc Edinb. 1919;52: 399-433. doi:10.1017/S0080456800012163

2. Wright S. Evolution in Mendelian Populations. Genetics. 1931;16: 97-159.

3. Ma Y, Ding X, Qanbari S, Weigend S, Zhang Q, Simianer H. Properties of different selection signature statistics and a new strategy for combining them. Heredity. 2015;115: 426-436. doi:10.1038/hdy.2015.42

4. Beissinger T, Kruppa J, Cavero D, Ha N-T, Erbe M, Simianer H. A Simple Test Identifies Selection on Complex Traits. Genetics. 2018;209: 321-333. doi:10.1534/genetics.118.300857

5. Lorenz AJ, Beissinger TM, Silva RR, de Leon N. Selection for Silage Yield and Composition Did Not Affect Genomic Diversity Within the Wisconsin Quality Synthetic Maize Population. G3 GenesGenomesGenetics. 2015;5: 541-549. doi:10.1534/g3.114.015263

6. Wood AR, Esko T, Yang J, Vedantam S, Pers TH, Gustafsson S, et al. Defining the role of common variation in the genomic and biological architecture of adult human height. Nat Genet. 2014;46: 1173-1186. doi:10.1038/ng.3097

7. Visscher PM, Hemani G, Vinkhuyzen AAE, Chen G-B, Lee SH, Wray NR, et al. Statistical Power to Detect Genetic (Co)Variance of Complex Traits Using SNP Data in Unrelated Samples. PLOS Genet. 2014;10: e1004269. doi:10.1371/journal.pgen.1004269

8. Visscher PM, Wray NR, Zhang Q, Sklar P, McCarthy MI, Brown MA, et al. 10 Years of GWAS Discovery: Biology, Function, and Translation. Am J Hum Genet. 2017;101: 5-22. doi:10.1016/j.ajhg.2017.06.005

9. Pritchard JK, Di Rienzo A. Adaptation - not by sweeps alone. Nat Rev Genet. 2010;11: 665-667. doi:10.1038/nrg2880

10. Pritchard JK, Pickrell JK, Coop G. The Genetics of Human Adaptation: Hard Sweeps, Soft Sweeps, and Polygenic Adaptation. Curr Biol. 2010;20: R208-R215. doi:10.1016/j.cub.2009.11.055

11. Hancock AM, Alkorta-Aranburu G, Witonsky DB, Di Rienzo A. Adaptations to new environments in humans: the role of subtle allele frequency shifts. Philos Trans R Soc $B$ Biol Sci. 2010;365: 2459-2468. doi:10.1098/rstb.2010.0032

12. Berg JJ, Coop G. A Population Genetic Signal of Polygenic Adaptation. W. Feldman M, editor. PLoS Genet. 2014;10: e1004412. doi:10.1371/journal.pgen.1004412 
13. Field Y, Boyle EA, Telis N, Gao Z, Gaulton KJ, Golan D, et al. Detection of human adaptation during the past 2000 years. Science. 2016;354: 760-764. doi:10.1126/science.aag0776

14. Csilléry K, Rodríguez-Verdugo A, Rellstab C, Guillaume F. Detecting the genomic signal of polygenic adaptation and the role of epistasis in evolution. Mol Ecol. 2018;27: 606612. doi:10.1111/mec.14499

15. Zeng J, Vlaming R de, Wu Y, Robinson MR, Lloyd-Jones LR, Yengo L, et al. Signatures of negative selection in the genetic architecture of human complex traits. Nat Genet. 2018;50: 746-753. doi:10.1038/s41588-018-0101-4

16. Sohail M, Maier RM, Ganna A, Bloemendal A, Martin AR, Turchin MC, et al. Polygenic adaptation on height is overestimated due to uncorrected stratification in genomewide association studies. Nordborg M, McCarthy MI, Nordborg M, Barton NH, Hermisson J, editors. eLife. 2019;8: e39702. doi:10.7554/eLife.39702

17. Berg JJ, Harpak A, Sinnott-Armstrong N, Joergensen AM, Mostafavi H, Field $Y$, et al. Reduced signal for polygenic adaptation of height in UK Biobank. Nordborg $M$, McCarthy MI, Nordborg M, Barton NH, Hermisson J, editors. eLife. 2019;8: e39725. doi:10.7554/eLife.39725

18. Lloyd-Jones LR, Zeng J, Sidorenko J, Yengo L, Moser G, Kemper KE, et al. Improved polygenic prediction by Bayesian multiple regression on summary statistics. Nat Commun. 2019;10: 1-11. doi:10.1038/s41467-019-12653-0

19. Erbe M, Hayes BJ, Matukumalli LK, Goswami S, Bowman PJ, Reich CM, et al. Improving accuracy of genomic predictions within and between dairy cattle breeds with imputed high-density single nucleotide polymorphism panels. J Dairy Sci. 2012;95: 4114-4129. doi:10.3168/jds.2011-5019

20. Josephs EB, Berg JJ, Ross-Ibarra J, Coop G. Detecting Adaptive Differentiation in Structured Populations with Genomic Data and Common Gardens. Genetics. 2019;211: 989-1004. doi:10.1534/genetics.118.301786

21. Beissinger $T$, Kruppa J, Cavero D, Ha N-T, Erbe M, Simianer H. A Simple Test Identifies Selection on Complex Traits. Genetics. 2018;209: 321-333. doi:10.1534/genetics.118.300857

22. R: The R Project for Statistical Computing. [cited 2 Jan 2020]. Available: https://www.rproject.org/

23. Beissinger T. timbeissinger/ComplexSelection. 2019. Available: https://github.com/timbeissinger/ComplexSelection

24. Voss-Fels KP, Stahl A, Wittkop B, Lichthardt C, Nagler S, Rose T, et al. Breeding improves wheat productivity under contrasting agrochemical input levels. Nat Plants. 2019;5: 706-714. doi:10.1038/s41477-019-0445-5 
25. Ooi H, Corporation M, Weston S, Tenenbaum D. doParallel: Foreach Parallel Adaptor for the "parallel" Package. 2019. Available: https://CRAN.Rproject.org/package=doParallel

26. Endelman JB. Ridge Regression and Other Kernels for Genomic Selection with R Package rrBLUP. Plant Genome. 2011;4: 250-255. doi:10.3835/plantgenome2011.08.0024

27. Pasaniuc $B$, Price $A L$. Dissecting the genetics of complex traits using summary association statistics. Nat Rev Genet. 2017;18: 117-127. doi:10.1038/nrg.2016.142

28. Ganal MW, Durstewitz G, Polley A, Bérard A, Buckler ES, Charcosset A, et al. A Large Maize (Zea mays L.) SNP Genotyping Array: Development and Germplasm Genotyping, and Genetic Mapping to Compare with the B73 Reference Genome. PLOS ONE. 2011;6: e28334. doi:10.1371/journal.pone.0028334

29. Muqaddasi QH, Brassac J, Börner A, Pillen K, Röder MS. Genetic Architecture of Anther Extrusion in Spring and Winter Wheat. Front Plant Sci. 2017;8.

doi:10.3389/fpls.2017.00754

30. Benesty J, Chen J, Huang Y, Cohen I. Pearson Correlation Coefficient. In: Cohen I, Huang $Y$, Chen J, Benesty J, editors. Noise Reduction in Speech Processing. Berlin, Heidelberg: Springer; 2009. pp. 1-4. doi:10.1007/978-3-642-00296-0_5

31. Avgar T, Lele SR, Keim JL, Boyce MS. Relative Selection Strength: Quantifying effect size in habitat- and step-selection inference. Ecol Evol. 2017;7: 5322-5330. doi:10.1002/ece3.3122

32. Coleman ML, Chisholm SW. Ecosystem-specific selection pressures revealed through comparative population genomics. Proc Natl Acad Sci. 2010;107: 18634-18639. doi:10.1073/pnas.1009480107

33. Burton PR, Clayton DG, Cardon LR, Craddock N, Deloukas P, Duncanson A, et al. Genome-wide association study of 14,000 cases of seven common diseases and 3,000 shared controls. Nature. 2007;447: 661-678. doi:10.1038/nature05911

34. Price AL, Patterson NJ, Plenge RM, Weinblatt ME, Shadick NA, Reich D. Principal components analysis corrects for stratification in genome-wide association studies. Nat Genet. 2006;38: 904-909. doi:10.1038/ng1847

35. Gengler N, Mayeres P, Szydlowski M. A simple method to approximate gene content in large pedigree populations: application to the myostatin gene in dual-purpose Belgian Blue cattle. animal. 2007;1: 21-28. doi:10.1017/S1751731107392628 


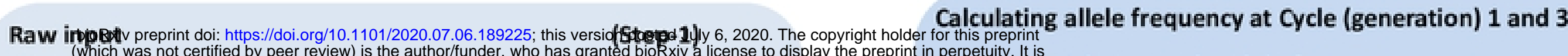

\#Install Ghat package

install.packages ("Ghat")

\# Loading Ghat and the dependent packages

library (Ghat)

library (parallel)

library (rrBLUP)

\# Loading phenotypes, map, genotype data

\# that are available in Ghat package

phe $\quad<-$ Maize_wqs [ [2]]

map <- Maize_wqs [ [3]]

gen <- Maize_wqs [ [4]]

\# Runing rrBLup to estimate all markers effects,

\# using full maximum-likelihood method ("ML")

result <- mixed.solve (phe [,2],

$\mathrm{Z}=$ as.matrix $(\operatorname{gen}[, 2: \operatorname{ncol}($ gen $)])$,

$\mathrm{X}=$ model.matrix (phe $[, 2] \sim$ phe $[, 3])$,

$\mathrm{K}=\mathrm{NULL}, \mathrm{SE}=\mathrm{FALSE}$,

return.Hinv=FALSE, method="ML")

\section{Calculating Ghat}

(Step-4)

\# Using effective number of independent marker

\# of 54.74819 (see step 3)

Ghat.adf <- Ghat (effects=result $\$$, change=change, method = "scale", perms $=1000$, plot="Ghat", num_eff $=54.74819$ )

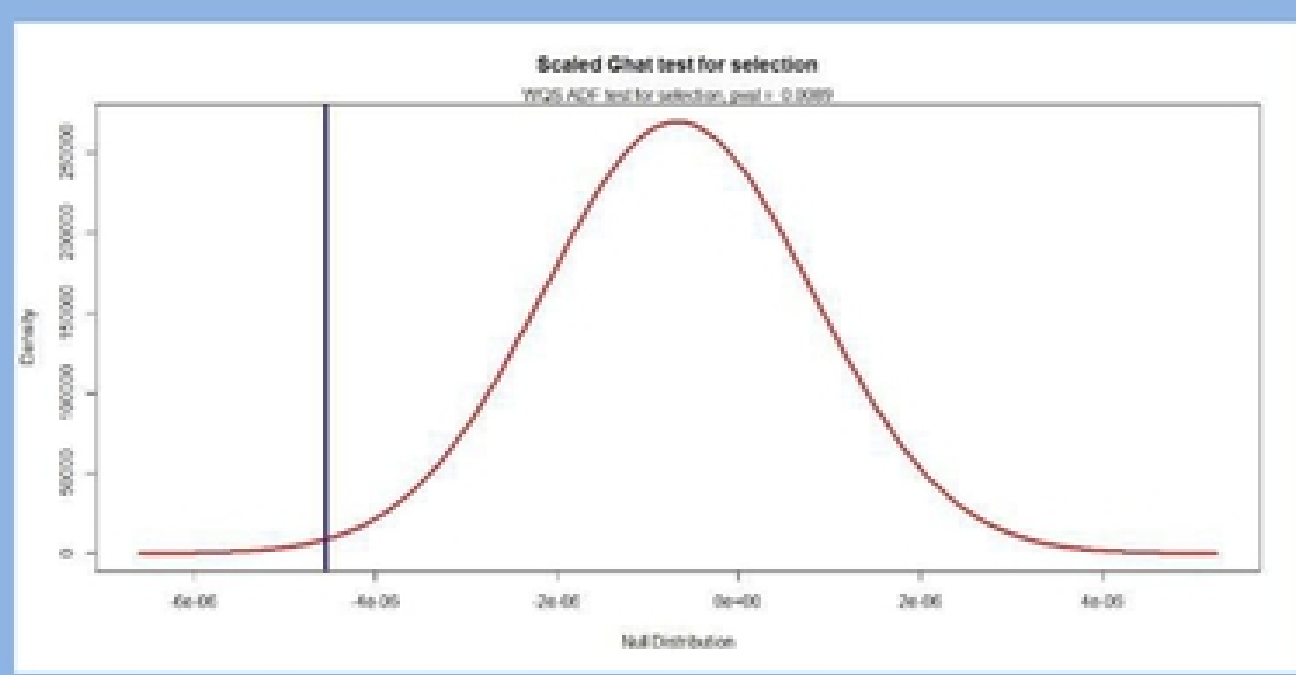

\# indicating each chicle

$\mathrm{Cy}_{-}$ind $<-$as.numeric (unlist (strsplit (gen $\$ \mathrm{X}$,

Cycle1 split="C")) [seg $\left(2,2{ }^{\star}\right.$ nrow $($ gen $\left.\left.\left.), 2\right)\right]\right)$

Cycle3 <- gen [which (Cy ind $==3)$, ]

CycleList $\quad<-$ list (Cycle1, $\bar{C}$ ycle3)

\# Calculating the frequencies

Frequencies <- matrix (nrow=ncol (gen) -

1 , $\mathrm{ncol}=2$ )

for (i in $1: 2)$ (

frequencies[,i] <- colMeans (CycleList [[i]] [, -

1], na.rm=TRUE) /2

\}

Frequencies <- as.data.frame (frequencies)

names (frequencies) <- c ("Cycle1", "Cycle3")

\# Calculating changes in allele frequencies from cycle 1 to cycle 3

Change<-frequencies $\$$ Cycle 3 -frequencies $\$$ Cycle 1

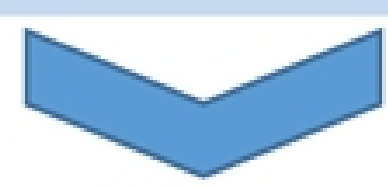

\section{Calculating Linkage disequilibrium (LD) Decay}

(Step- 3)

\# Using ld decay function that implemented in

\# Ghat, with a threshold of correlation between

\# neighbouring of 0.03

Ld

<- ld decay (gen=gen, map=map, $\max$ win $\mathrm{snp}=2000, \max . \operatorname{ch} r=10$, cores $=1$, max_r2=0.03)

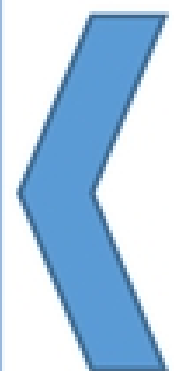

Number of SN.Ps in LO at Threshold of $<0.03$

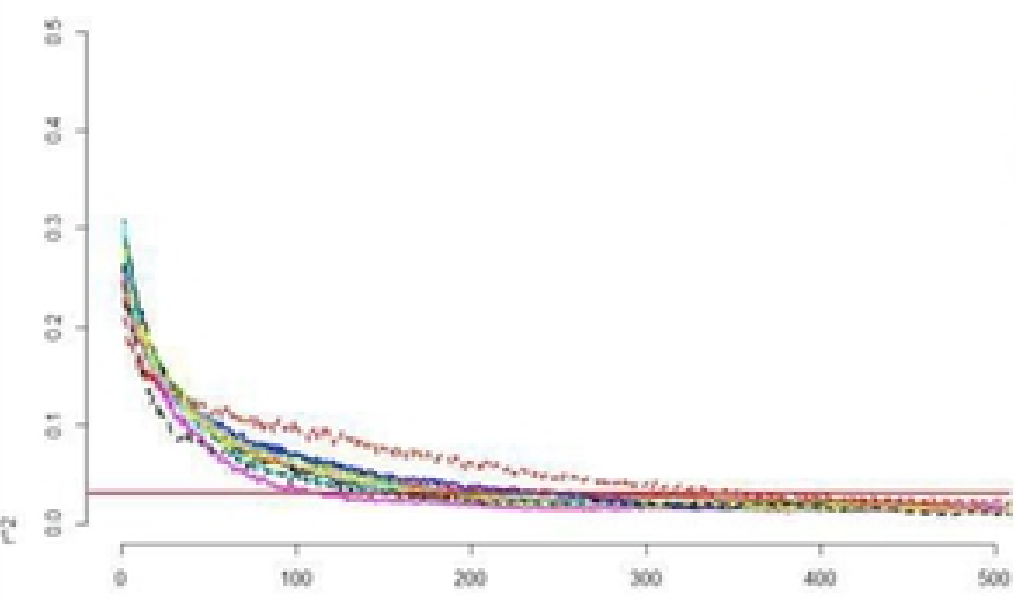

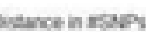


50 years of phenotypes 3 years of phenotypes

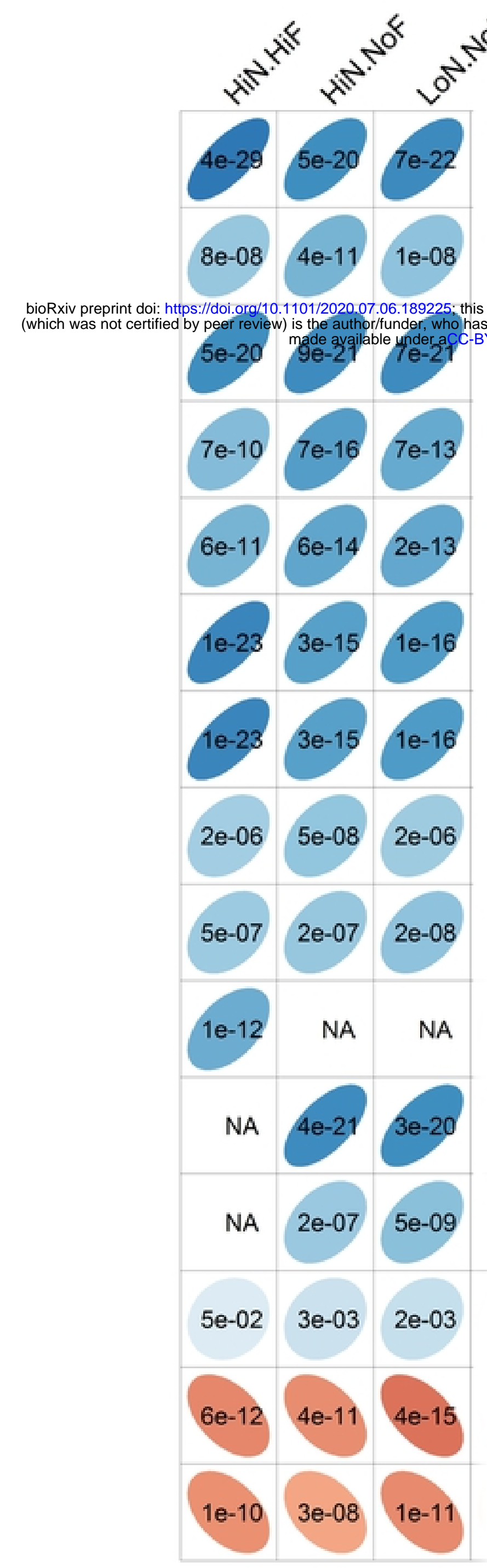

Above-ground plant biomass

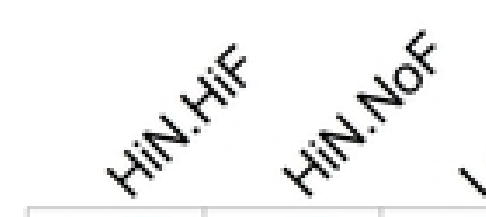

Grain yield

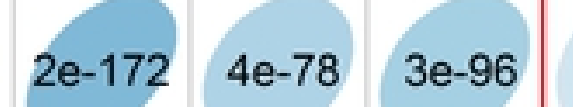

$6 e-3$
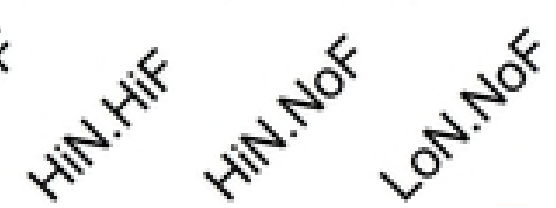

.

\section{6}

Harvest index

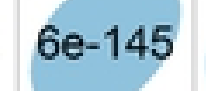

2

e-79

$2 e-155$

(6e-

Kernels per sqm

2e-124

$7 e-128$

5e-107

$2 e-11$

Kernel per spike

$3 e-110$

$3 e-104$

$3 e-108$

$6 e-14$

$2 e-09 \quad 7 e-11$

Protein Yield

2e-101

$6 e-54$

$5 e-67$

(

2e-91

6e-05

$1 e-13$

Nitrogen use efficiency

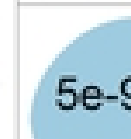

3

$-55$

$7 e-68$

$5 e-81$

$8 e-05 \quad 5 e-14$

Radiation use efficiency

$4 e-92$

$6 e-75$

$4 \mathrm{e}-94$

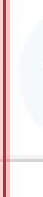

Radiation interception efficiency

3e-91

$2 e-83$

$2 e-89$

8e-

\begin{tabular}{|l|l|l|}
\hline $8 e-36$ & $2 e-05$ & $2 e-26$
\end{tabular}

Green canopy duration

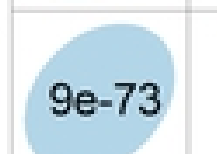

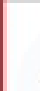

\begin{tabular}{|l|l|l|}
\hline $1 e-02$ & $3 e-01$ & $5 e-01$ \\
\hline $8 e-36$ & $2 e-05$ & $2 e-26$ \\
\hline
\end{tabular}

2

2e-01 NA NA

Resistance to powdery mildew

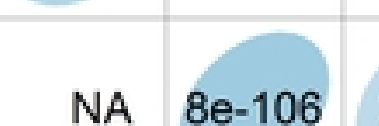

$2 e-58$

\begin{tabular}{l|l|l} 
NA & $7 \mathrm{e}-22$ & $4 \mathrm{e}-19$
\end{tabular}

\section{Resistance to stripe rust}

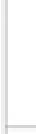

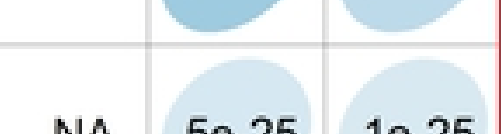

\begin{tabular}{l|l|l} 
NA & $9 \mathrm{e}-01$ & $2 \mathrm{e}-03$
\end{tabular}

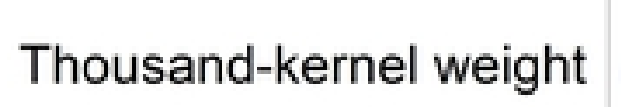

Plant height

$7 e-57$

$1 e-48$

$5 e-78$

$5 e-3$

Crude protein

$5 e-79$

$3 e-718 e-110$

\begin{tabular}{|l|l|l|}
\hline $4 e-05$ & $5 e-06$ & $5 e-09$
\end{tabular} 


\section{Cumulative Selection Pressure in Wheat}

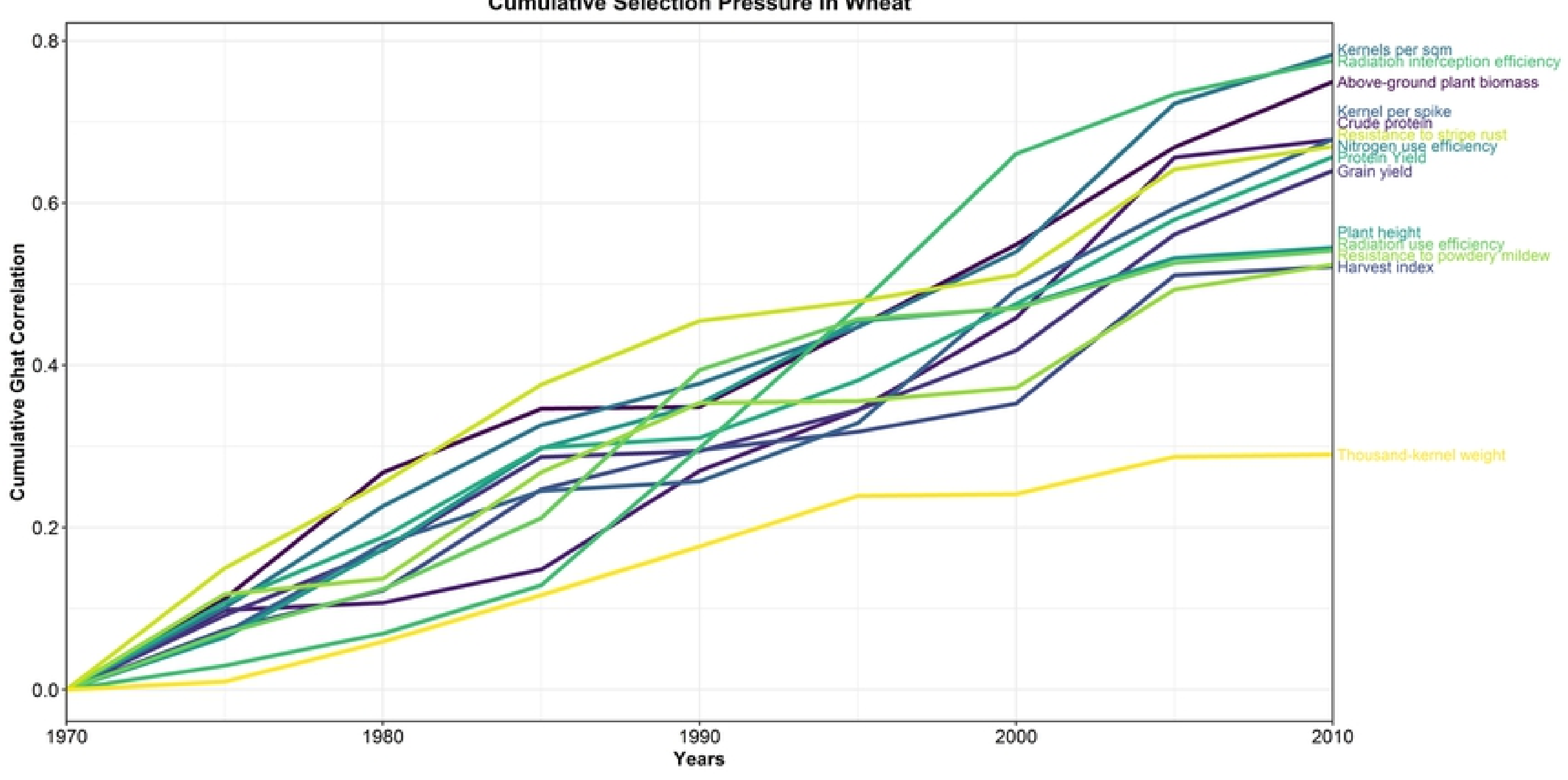

Fig4 